\title{
Development of high resolution camera for observations of superconducting cavities
}

\author{
Yoshihisa Iwashita and Yujiro Tajima* \\ Institute for Chemical Research, Kyoto University, Japan 611-0011
}

Hitoshi Hayano

High Energy Accelerator Research Organization (KEK), 1-1 Oho, Tsukuba, Ibaraki, Japan 305-0801

(Received 15 August 2008; published 16 September 2008)

\begin{abstract}
A system for inspecting the inner surface of superconducting rf cavities is developed in order to study the relation between the achievable field gradient and the defects in the inner surface. The inspection system consists of a high resolution complementary metal-oxide-semiconductor camera and a special illumination system built in a cylinder that has a diameter of $50 \mathrm{~mm}$. The camera cylinder can be inserted into the L-band 9 cell superconducting cavity. The system provides a resolution of about $7.5 \mu \mathrm{m} /$ pixel. Thus far, there have been good correlations between locations identified by thermometry measurements and positions of defects found by this system. The heights or depths of the defects can also be estimated by measuring wall gradients using the reflection angle relation between the camera position and the strip illumination position. This paper presents a detailed description of the system and the data obtained from it.
\end{abstract}

DOI: 10.1103/PhysRevSTAB.11.093501

PACS numbers: $29.20 .-\mathrm{c}$

\section{INTRODUCTION}

TESLA-type [1] superconducting (Sc) accelerating cavities have been in development for over ten years [2-6]. Increased accelerating field gradients have been obtained due to improvements in cavity fabrication processes. The yield of high gradient cavities, however, must be improved for applications such as the XFEL [7] and ILC [8]. The ILC requires more than 140009 -cell cavities with a gradient and yield exceeding $35 \mathrm{MV} / \mathrm{m}$ and $80 \%$, respectively. The main obstacles to achieving a high gradient are thought to be thermal breakdown (TB) and field emission (FE), supposedly caused by surface defects with sizes of the order $100 \mu \mathrm{m}$ and $1 \mu \mathrm{m}$ [2]. For thermal effects, simulations have been made which show that normal conducting defects in the equator region in the order of 30-50 $\mu \mathrm{m}$ would limit TESLA cavities with an RRR $=300$ to gradients of 20-25 MV/m [2]. For field emission the spot sizes that are calculated are typically very small $(<1 \mu \mathrm{m})$ but it has been experimentally shown that they reside on larger surface defects like scratches, etc. An optical inspection should be the first choice for finding such defects $[9,10]$. In order to find defects of the order of $100 \mu \mathrm{m}$ in size on the inner surface of a cavity, we developed a high resolution camera. The resolution of this camera is about $7.5 \mu \mathrm{m} /$ pixel, and objects that are more than several tens of microns in size can be observed with it. This system revealed undiscovered defects on the inner sides of the

\footnotetext{
*Present address: Toshiba Corporation, Yokohama, Kanagawa, Japan.
}

cavities predicted by pass-band-mode measurements and thermometry measurements.

\section{CAMERA SYSTEM}

\section{A. Camera cylinder}

The inner surfaces of Sc cavities are highly reflective; but they are not perfect mirrors. This makes lighting for optical inspection very difficult; uniform lighting provides an image that is flat and does not convey much information. Therefore, we use electroluminescence (EL) sheets for lighting. EL sheets have the advantages of a thin light source; they can be easily cut into various shapes and bent so that they can cover the outer surface of the camera cylinder. In order to carry out a nondestructive observation and keep the inner surface as clean as possible, the camera system is housed in a cylinder with a diameter of $50 \mathrm{~mm}$ (see Fig. 1). This cylinder can be inserted into the bores of L-band 9-cell cavities, because nothing protrudes from it. The advantage of placing the camera in a housing is that it eliminates the possibility of scratching the inner surface of

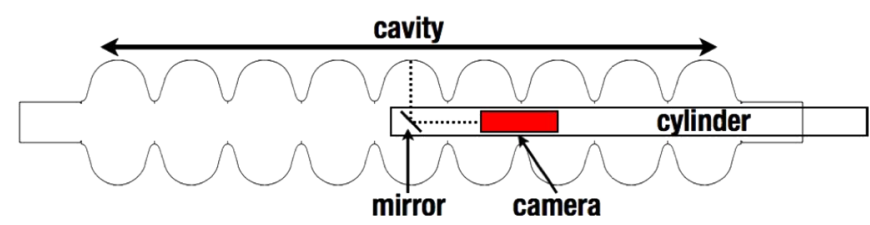

FIG. 1. (Color) Schematic diagram of our cavity inspection system. The cavity swallows the camera cylinder by moving longitudinally. The cylinder does not move. The inner surface reflected in an imaging mirror is observed. The mirror is located in front of the camera. 


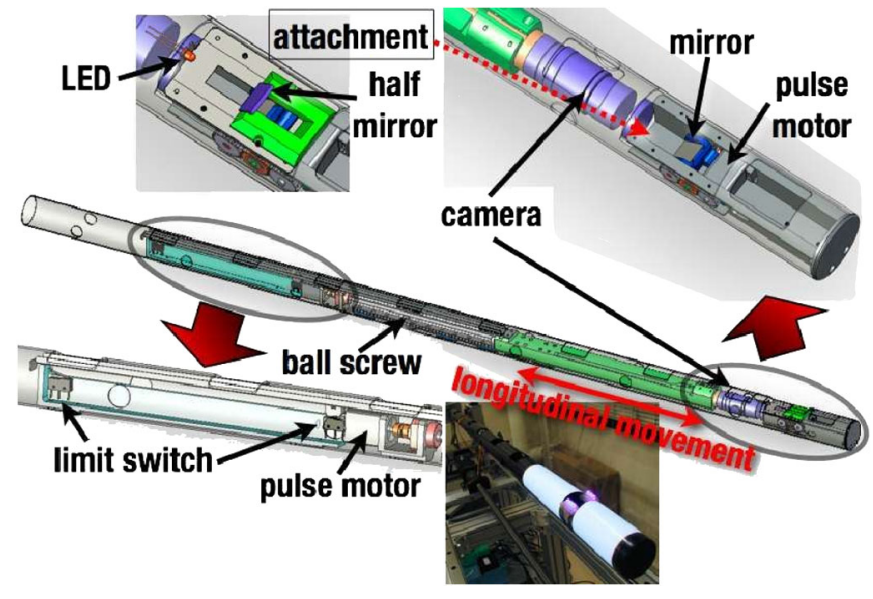

FIG. 2. (Color) Inside of the camera cylinder. The inset shown in upper right corner shows the mirror and its drive PM. The camera moves for focal adjustment. The EL sheets for the illumination are shown at the bottom.

the cavity due to camera movement. The camera is installed in the cylinder and is trained on the head of the cylinder where a mirror reflects the inner surface of the cavity. The imaging mirror can be tilted by a pulse motor (PM) to show a surface other than the cavity equator. The focus is adjusted by motorized positioning of the camera that keeps the optical length (working distance) between the object and the camera lens constant (see Fig. 2). The camera used in this system is a 1.5 megapixel three-layered complementary metal-oxide-semiconductor color camera (CSF5M7C3L18NR, Toshiba-Teli), whose pixel size is $5 \times 5 \mu \mathrm{m}$. The camera is equipped with a c-mount for the lens interface. A low distortion lens (VS-LD75, V. S. Technology Corp.) with extension tubes installed between the lens and the camera gives a maximum magnification factor of 0.7. Thus, the maximum resolution at the object surface corresponds to $7.5 \mu \mathrm{m} /$ pixel. Because one frame covers an area of approximately $10 \mathrm{~mm} \times 7 \mathrm{~mm}$, at least 80 shots are required to record the whole $10 \mathrm{~mm}$ band region around the single electron beam welding (EBW) seam on an equator. The file size of the captured frame is about $5 \mathrm{MB}$. Thus, the total storage size needed to record images at regions around the equator of the cavity is $80 \times$ $9 \times 5 \mathrm{MB}=3.6 \mathrm{~GB}$. Data of this size can fit in a DVD-R.

Illumination is provided by EL sheets that are mounted on the surface of the cylinder. Because EL sheets are not transparent, the camera requires a viewing window to look though. We divided an EL sheet at the center to make the viewing window. They are further divided into stripes, and each stripe has an independent on/off control switch in order to change the position of illumination. This feature is useful for evaluating irregularities, as explained later. A surface perpendicular to the optical axis can be observed by coaxial illumination. In this technique, a half mirror with an LED lamp is placed between the lens head and the imaging mirror, without interfering the camera view. When

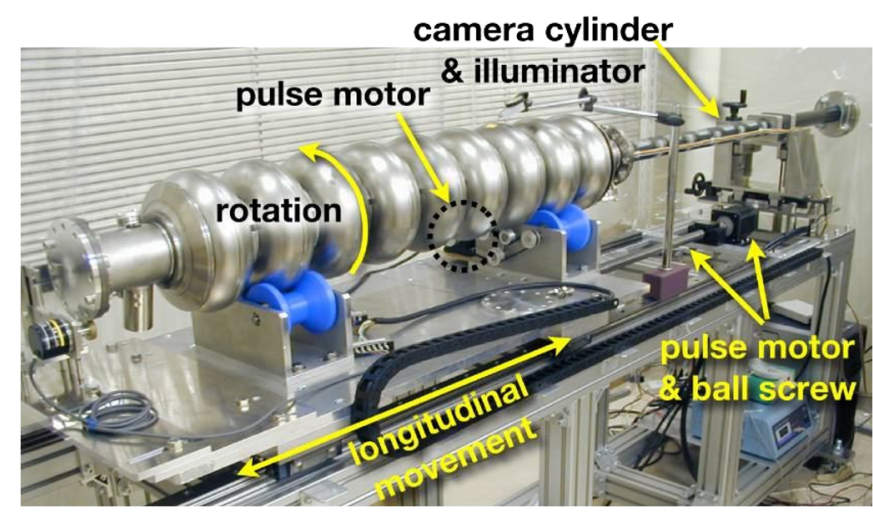

FIG. 3. (Color) Overview of our inspection system.

we need to observe locations other than equators and irises, the imaging mirror must be tilted. As the depth of field is very narrow (about $0.1 \mathrm{~mm}$ ), the line of sight has to be close to the surface normal. This illumination method works at any tilt angle of the mirror, since the lighting axis is always on the line of sight, while the EL sheets have to be slid in order to locate the viewing window on the optical axis.

The brightness of both the EL sheets and LED can be adjusted by an illumination controller-frequency change for EL sheets and current control for the LED.

\section{B. Cavity positioning table}

A cavity can be slid in the longitudinal direction and rotated around its axis, while the position of the camera cylinder is fixed so that the inner surface of every cell can be observed (see Fig. 3). The longitudinal position of the cavity is determined by the number of pulses fed to the PM, while the azimuthal coordinates are measured by the rotary encoder installed at the edge of a cavity flange. Both the longitudinal and azimuthal positioning resolutions are less than $5 \mu \mathrm{m}$, which should be enough for our purpose. The step resolution of the PM can be increased, using a special function of pulse motor controllers.

Although the camera cylinder is fixed on the table, the movement of the cavity during its positioning shakes the cylinder. This disturbs the observation during the swing because of the camera's very narrow depth of field. A damper installed at the end of the cylinder helps to damp the swing quickly.

\section{OBSERVATIONS}

\section{A. Zanon 84}

The first cavity that was observed by the camera system was Z84 borrowed from DESY. While this cavity was diagnosed as Q-diseased at DESY, a gradient of $27 \mathrm{MV} / \mathrm{m}$ was recorded by passband-mode measurement. The $10 \mathrm{~mm}$ band regions on the equators were observed at a resolution of $15 \mu \mathrm{m} /$ pixel. Figure 4 shows a typical 


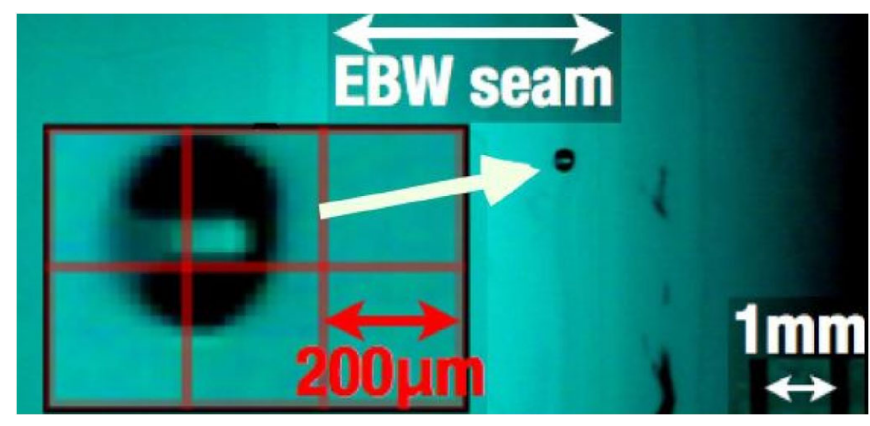

FIG. 4. (Color) Typical image of Z84. Many cat's-eye-shaped spots were observed.

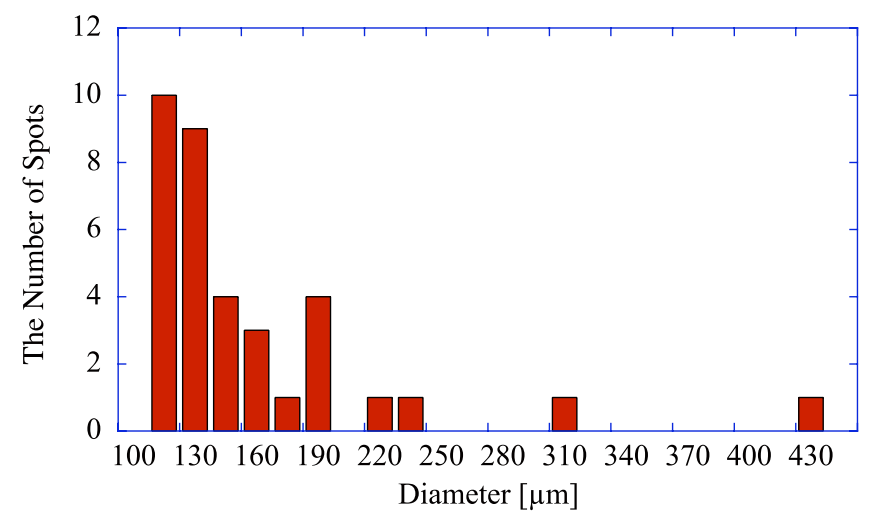

FIG. 5. (Color) Population of diameter from Z84 observation.

image obtained by the system. There were 35 spots whose diameters were more than $100 \mu \mathrm{m}$. The population distribution shown in Fig. 5 indicates the existence of many spots with smaller diameters. It should be mentioned that these spots are not just on the EBW seam center but also on the seam edge of the input coupler side.

\section{B. AES001}

AES001 from FNAL was the second cavity, whose surface was not barrel polished. It showed a quench at $15 \mathrm{MV} / \mathrm{m}$ by $\mathrm{TB}$, while there was no FE [11-13]. The quenched cells identified by the passband-mode measurement were cell 3 and/or cell 7. A thermometry measurement further localized the hot spot positions at the equator regions in cell 3 (see Fig. 6, left), and two hot spot locations at the thermosensors of cells 4 and 5 were identified. We inserted the camera and observed the inner surfaces at these locations with a resolution of about $7 \mu \mathrm{m} /$ pixel and found defects that were cat's-eye shaped at locations predicted by the thermometry measurements (see Fig. 6, right). The locations can be identified as follows: (i) place a pointer at the center of the camera view (off-cavity, see Fig. 7, top figures), (ii) position the cavity such that it is aimed at the defect (see Fig. 7, bottom right), and (iii) check the location on the cavity pointed by the pointer (see Fig. 7, bottom left). Hot spot \#5 had twin defects that were about $600 \mu \mathrm{m}$ in diameter, and hot spot \#4 had a defect that was about $400 \mu \mathrm{m}$ in diameter. Figure 8 shows a detailed image of the latter spot in scale along with a few notes. Although these three spots have a similar shape, that is, a cat's eye, the pupils (bars) in the twins are not clear, maybe because of contaminations.

We also found defects in the equator region of cell 7 , which was predicted by the passband-mode measurement as a source of TB, as shown in Fig. 9. Twins are also found in cell 3. It should be noted that these defects are found at the edges of EBW seams (including Z84 case). These locations are heat-affected regions that lie between the completely melted region and the original region where the grain size changes. These were the only defects found in AES001. However, some smears, scratched, and arc scars were observed.

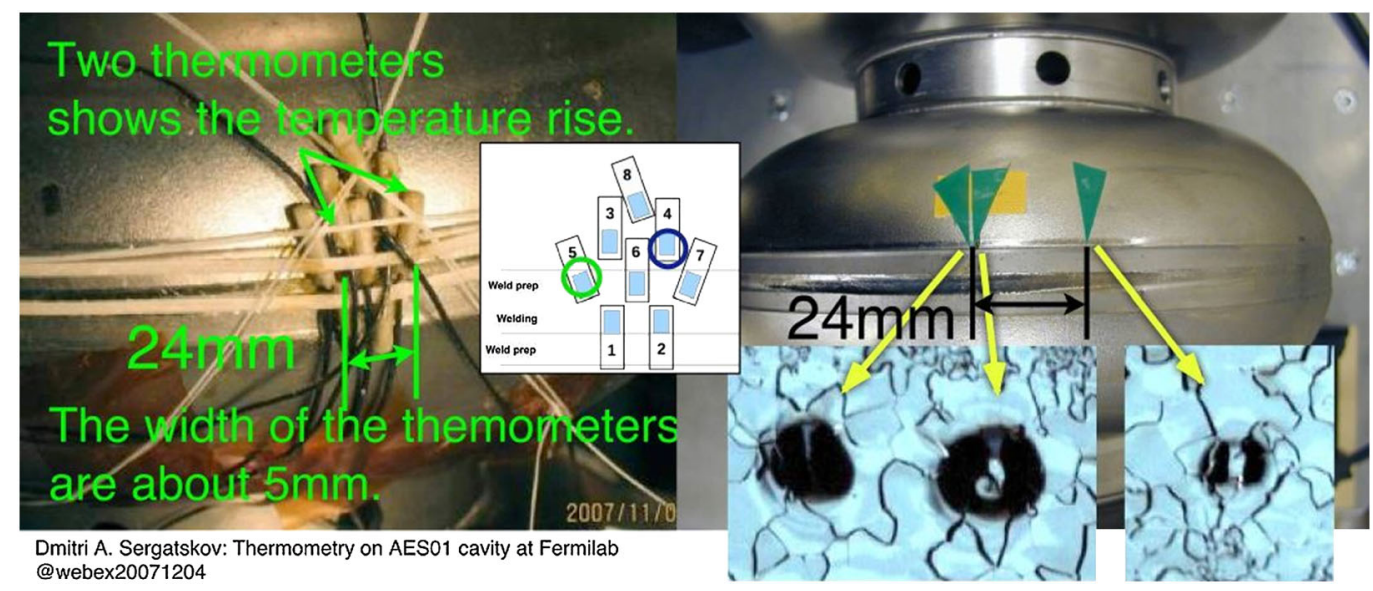

FIG. 6. (Color) Positions of the two hot spots found at FNAL (left) and those found at Kyoto (right). The inset of the left figure shows the location of the thermometers. The two thermometers (\#4 and \#5) that showed an abnormal temperature rise are marked. (Courtesy of FNAL/JLAB.) 


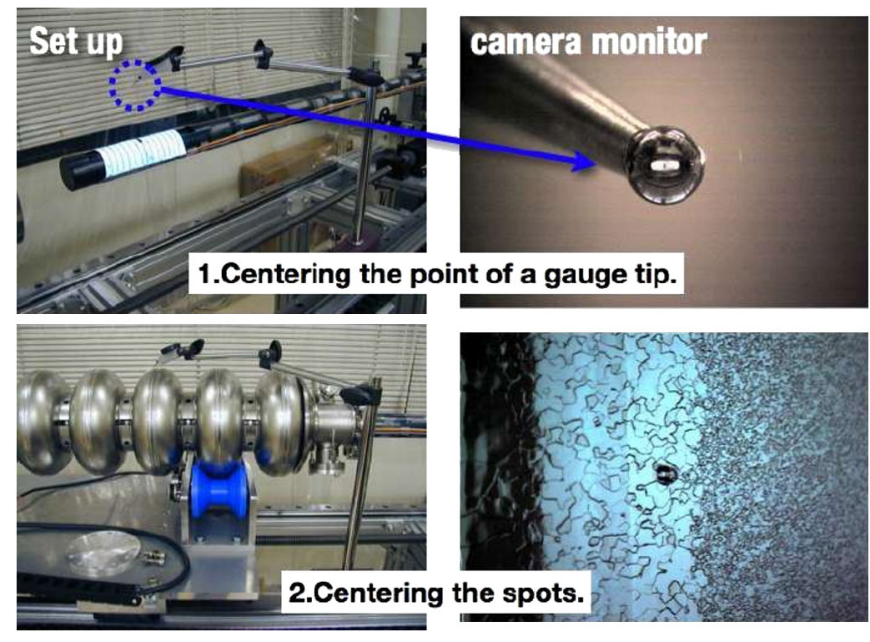

FIG. 7. (Color) Pointer to confirm the position monitored by the camera on the outer wall of a cavity.

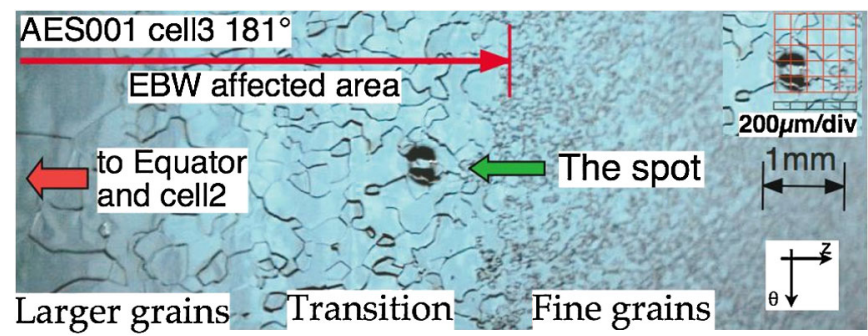

FIG. 8. (Color) Cat's-eye spot found at the equator region of cell $3,181^{\circ}$. The diameter is about $400 \mu \mathrm{m}$.

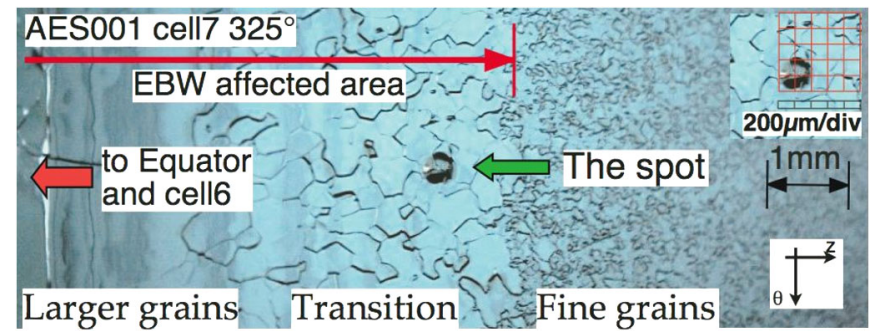

FIG. 9. (Color) Cat's-eye spot found at the equator region of cell $7,325^{\circ}$. The diameter is about $400 \mu \mathrm{m}$.

\section{HEIGHT MEASUREMENT}

The camera images show only the cross section of the defects; no height information can be obtained from these images. A wall gradient of the inner surface of a cavity can be estimated if the position of illumination is known.

Figure 10 shows the principle of the wall gradient measurement [14]. The rotational axis of a cavity is $z$. Let the angle between the $z$ axis and the mirror located in front of the camera be $45^{\circ}$, for simplicity. Suppose that we observe a point on an inner surface of the cavity $(z, r)=(0, R)$. The angle between the tangential line at a point on the inner

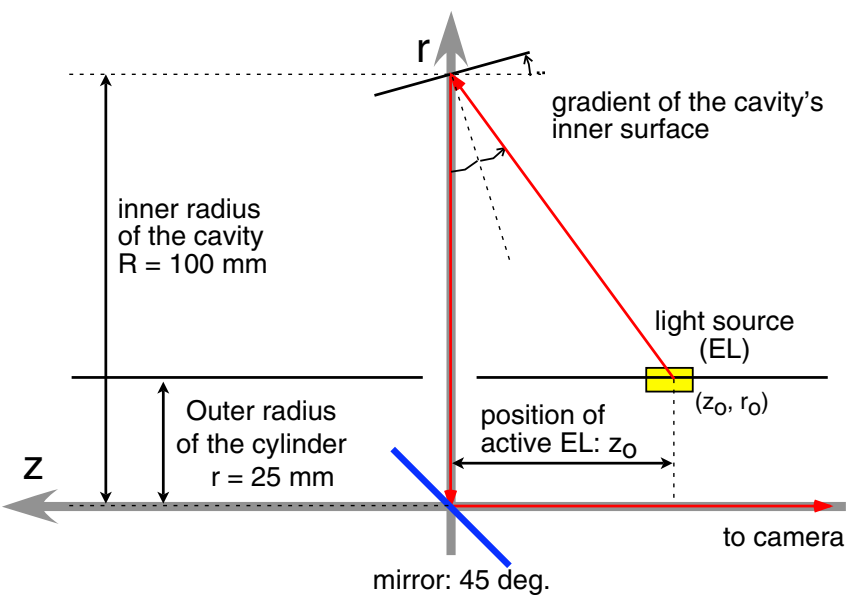

FIG. 10. (Color) Schematic drawing of the wall gradient measurement. The wall gradient, the incident angle and the reflection angle are the same. The value $\theta$ can be calculated from the position $z_{o}$ of the illuminator.

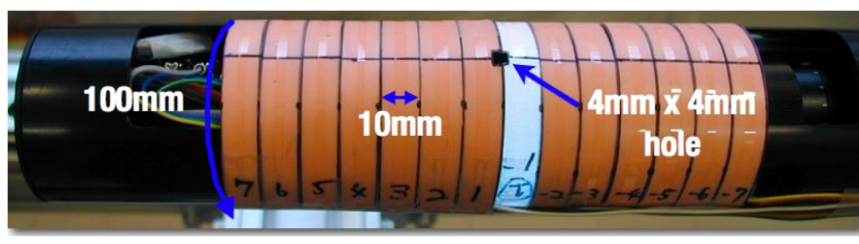

FIG. 11. (Color) Stripe illuminator. The mirror is located under the hole between the two stripes numbered \#1 and -1 . The 14 stripes can be independently turned ON/OFF.

surface of the cavity and the $z$ axis, that is, the wall gradient, is $\theta$. When the incident angle of light from the point is $\theta$, the light is reflected into the camera and the observed point is illuminated to form an image. Then, from the point of the light source, $\left(z_{o}, r_{o}\right)$, the wall gradient $\theta$ is given by

$$
\theta=\frac{1}{2} \arctan \frac{z_{o}}{R-r_{o}}
$$

In order to carry out the wall gradient measurement, the EL sheets are further divided into stripes to form a stripe illuminator, as shown in Fig. 11, where each EL strip works independently. When the light strip band on the EL sheet runs along the axis, a bright point moves in a cat's-eye pupil. Thus, from the available angle information, we can estimate the wall gradient as the tilt angle of the mirror surface. Figure 12 shows an example of a convex spot. The black curve in the left figure is the fitted differential Gaussian, from which the height of the spot is estimated as $40 \mu \mathrm{m}$. The black curve in the right figure is the fitted Gaussian, from which the height of the spot is calculated as $42 \mu \mathrm{m}$. Integration of a wall gradient along a line gives the height profile of a defect. Figure 13 shows an example of a concave spot found at cell 7 . Again, the black curve in the 

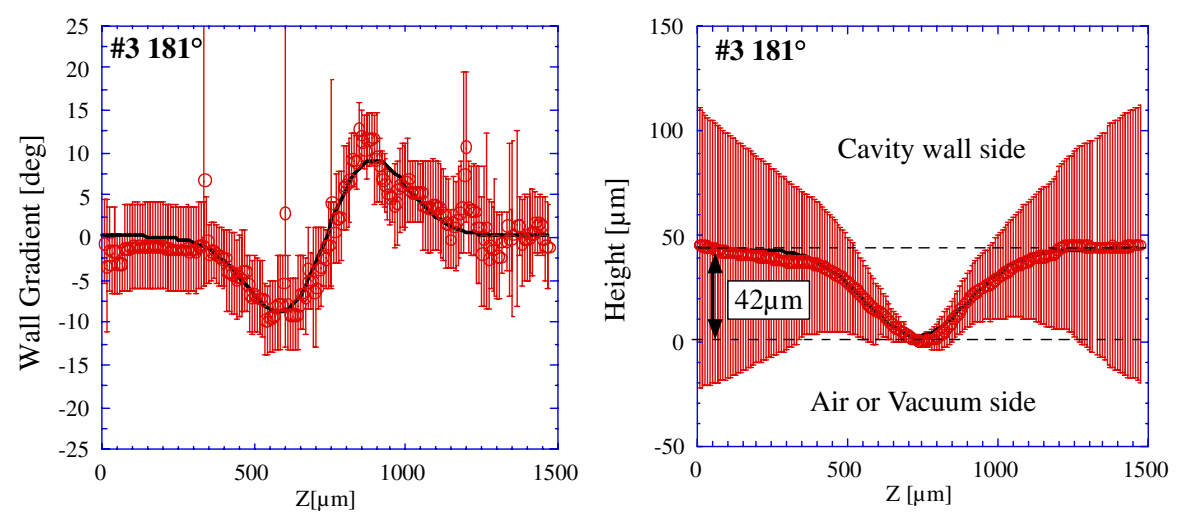

FIG. 12. (Color) Gradient and height of the spot shown in Fig. 8. The spot is convex. The height is expressed by a relative value of the distance from the cavity axis.
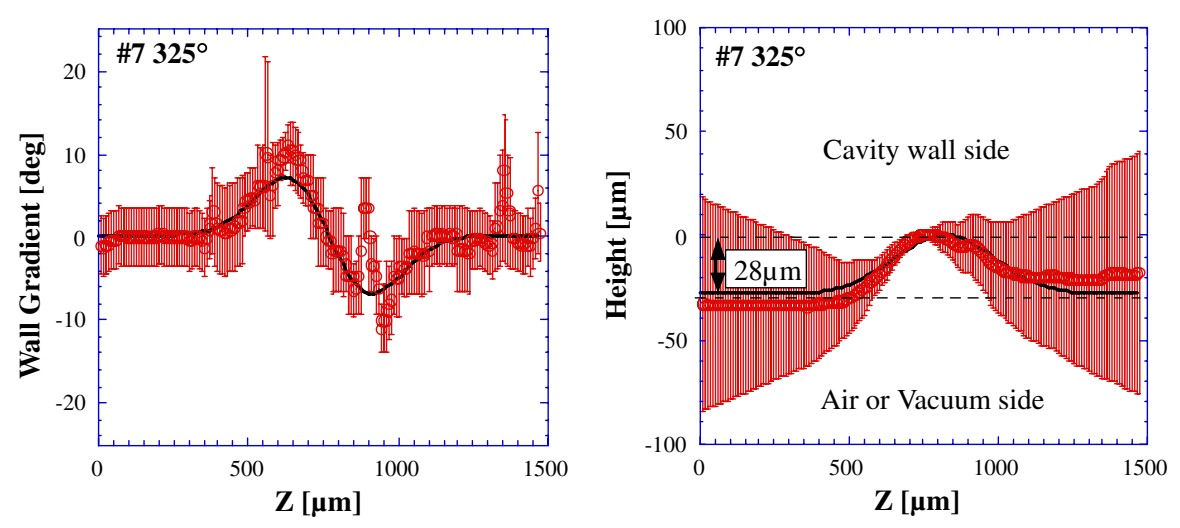

FIG. 13. (Color) Gradient and height of the spot shown in Fig. 9. This example shows a concave spot (pit).

left figure is the fitted differential Gaussian, from which the height of the spot is obtained as $29 \mu \mathrm{m}$. The black curve in the right figure is the fitted Gaussian, from which the height of the spot is obtained as $28 \mu \mathrm{m}$.

\section{DISCUSSIONS}

The spots we found tend to be located in the heataffected regions. Understanding the causes of the localized defects would help us to improve the yield and performance of the cavities. Further investigations, such as chemical analysis and recrystallization process, are needed for this purpose. These may also improve reliabilities of general electron welding techniques.

The height measurement technique using the stripe illuminator helps in understanding not only the cause of the defects on the surface but also its effect on the accelerating gradient. Some spots do not show all the gradient information along the pupils (they are dark) maybe because of local surface contamination or roughness. This may indicate the existence of an impurity in $\mathrm{Nb}$ or contamination such as sulfur after electrochemical polishing.

The good correlation between the locations of defects identified by thermometry and the locations of defects found by the system can help in increasing the production yield of the cavity with a high gradient. After the discovery of the correlations, optical inspection with a high resolution camera is considered as an important process in the improvement of the cavity performance. This system is ready to be delivered to laboratories worldwide for improving cavity fabrication processes and their yields. Further improvements are under trial, such as higher resolution and easy manipulations.

\section{ACKNOWLEDGMENTS}

We thank all collaborators who helped us to achieve this work, especially Dr. L. Lilje at DESY, Dr. Shekhar Mishra, Dr. Bob Kephart, Dr. Mark Champion, Dr. C. M. Ginsburg, and Dr. D. A. Sergatskov at FNAL, and Dr. R. L. Geng at JLAB. The authors also thank Dr. Y. Kamiya and Dr. Y. Yokoya at KEK for funding this work.

[1] TeV-Energy Superconducting Linear Accelerator, TESLA Technical Design Report, Hamburg: Deutsches Elektronen-Synchrotron DESY, 2001, http://flash.desy. de/tesla/tesla_documentation. 
[2] H. Padamsee, J. Knobloch, and T. Hays, $R F$ Superconductivity for Accelerators (John Wiley, New York, 1998).

[3] H. Padamsee, IEEE Trans. Appl. Supercond. 15, 2432 (2005).

[4] H. Hayano, in Proceedings of the 4th Annual Meeting of Particle Accelerator Society of Japan and the 32nd Linear Accelerator Meeting in Japan, 2007, (Particle Accelerator Society of Japan, Tokyo, 2007), p. 218, http:// www.pasj.jp/web_publish/pasj4_lam32/PASJ4-LAM32\% 20(D)/contents/PDF/WP/WP02.pdf.

[5] L. Lilje, in Proceedings of the 21st International Linac Conference, Gyeongju, Korea, 2002 (Pohang Accelerator Laboratory, Pohang, Korea, 2002), p. 219.

[6] J. Knobloch, IEEE Trans. Appl. Supercond. 9, 1016 (1999).

[7] X-ray Free Electron Laser, The Technical Design Report of the European XFEL, ISBN 978-3-935702-17-1, Hamburg: Deutsches Elektronen-Synchrotron DESY, 2006, http://xfel.desy.de/tdr.

[8] International Linear Collider, International Linear Collider Reference Design Report, Hamburg: Deutsches Elektronen-Synchrotron DESY, 2006, http://www. linearcollider.org/rdr/.

[9] K. Saito, in Proceedings of the 21st Linear Accelerator Meeting in Japan, 1996 (Nihon University/Atomic Energy Research Institute, Tokyo, Japan, 1996), p. 219, in Japanese.

[10] T. Harden, M. Borden, A. Canabal, P. Pittman, and T. Tajima, in Proceedings of the 2007 Particle Accelerator
Conference, Albuquerque, New Mexico, 2007 (IEEE, Albuquerque, New Mexico, 2007), p. 2409, http:// cern.ch/AccelConf/p07/PAPERS/WEPMS032.PDF.

[11] C. M. Ginsburg, R. Carcagno, M. Champion, N. Dhanaraj, A. Lunin, A. Mukherjee, R. Nehring, D. Orris, J. Ozelis, V. Poloubotko, D. A. Sergatskov, and W.-D. Moeller, in Proceedings of the 13th International, Workshop on RF Superconductivity, Peking University, Beijing, China, 2007 (Institute of Heavy Ion Physics, Peking University, Beijing, 2007), http://www.pku.edu.cn/ academic/srf2007/download/proceedings/TUP47.pdf.

[12] R. Carcagno, C. M. Ginsburg, Y. Huang, R. Nehring, B. Norris, V. Poloubotko, R. Rabehl, I. Rakhno, C. Reid, T. Peterson, D. A. Sergatskov, C. Sylvester, M. Wong, C. Worel, A. Yuan, C. Grenoble, and T. J. Powers, in Proceedings of the 13th International Workshop on RF Superconductivity, Peking University, Beijing, China, 2007, Ref. [11], http://www.pku.edu.cn/academic/ srf2007/download/proceedings/WEP15.pdf.

[13] R. L. Geng, R. Afanador, A.C. Crawford, K. Davis, D. Forehand, C. Dreyfus, C. Grenoble, B. Golden, R. Johnson, P. Kushnick, K. Macha, J. Mammosser, J. Saunders, A. Wu, D. Bice, and D. A. Sergatskov, in Proc. 13th International Workshop on RF Superconductivity, Peking University, Beijing, China, 2007, Ref. [11], http://www.pku.edu.cn/academic/ srf2007/download/proceedings/WEP28.pdf.

[14] Y. Tajima, Master's thesis, Department of Physics, Kyoto University, 2007, in Japanese. 